NASA Technical Memorandum 101308

ICOMP-88-16

\title{
Least-Squares Finite Elements for Stokes Problem
}

\author{
(NASA-In-10.1308) LEAST-SCOAEES FIUITE \\ HEREATS RCB STCKES PECELEH, (HAL. Levis \\ Eesearch Center) $17 \mathrm{~F}$ CSCI $12 \mathrm{~A}$
}

$889-22392$

Unclas

G3/64 01988C4

Bo-Nan Jiang

Institute for Comptutational Mechanics in Propulsion

Lewis Research Center

Cleveland, Ohio

and

C.L. Chang

Cleveland State University

Cleveland, Ohio

December 1988

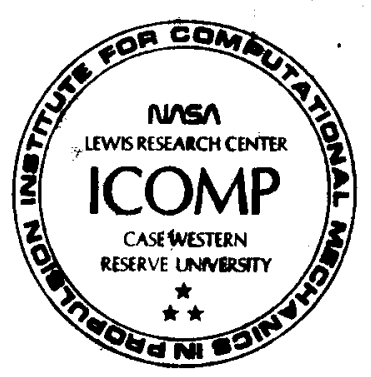




\title{
LEAST-SQUARES FINITE ELEMENTS FOR STOKES PROBLEM
}

\author{
Bo-Nan Jiang* \\ Institute for Computational Mechanics in Propulsion \\ Lewis Research Center \\ Cleveland, Ohio 44135
}

and

C.L. Chang

Department of Mathematics

Cleveland State University

Cleveland, Ohio 44115

\begin{abstract}
SUMMARY
A least-squares method based on the first-order velocity-pressurevorticity formulation for the Stokes problem is proposed. This method leads to a minimization problem rather than to a saddle-point problem. The choice of the combinations of elements is thus not subject to the Ladyzhenskaya-BabuskaBrezzi (LBB) condition. Numerical results are given for the optimal rate of convergence for equal-order interpolations.
\end{abstract}

\section{INTRODUCTION}

The Stokes problem has counterparts in many branches of engineering and physics. The Stokes operator is a basic ingredient of more complicated models of physical phenomena such as the incompressible Navier-Stokes equations. Any good solver of Navier-Stokes equations should at least be able to solve the Stokes equations. For this reason, the Stokes equations have been a focal point of finite element research for over 20 years.

For the Stokes flow, the Galerkin mixed method of velocity-pressure formulation is widely used. However, the mixed method leads to a saddle-point problem. Consequently, the combination of velocity and pressure interpolations is required to satisfy the Ladyzhenskaya-Babuška-Brezzi (LBB) condition (refs. 1 to 3), which precludes the application of many seemingly natural pairs of velocity and pressure elements. Although various convergent combinations of velocity and pressure elements have been developed, most of them are not convenient.

In attempting to overcome this difficulty, Hughes and his colleagues (ref. 4) introduced the Petrov-Galerkin method. They put an additional leastsquares term into the classical Galerkin mixed formulation in order to circumvent the LBB test and to apply an equal-order interpolation. Recently, Hughes et al. (ref. 5) have improved this formulation, and a symmetric matrix is attained.

*Work funded under Space Act Agreement C99066G. 
C.L. Chang, in a paper entitled "A Mixed Finite Element Method for Stokes Problem: Acceleration-Pressure Formulation," proposed a method employing equal-order interpolations for the Stokes problem. By changing variables and transforming the classical velocity-pressure formulation into two first-order systems with four and two variables, respectively, the least-squares method is applied to the systems sequentially. He has proved the optimal rate of convergence for both velocity and pressure by using Wendland's approach (ref. 6 ).

In this report we develop another least-squares method, a method based on the first-order velocity-pressure-vorticity formulation. The least-squares method leads to a minimization problem and thus is not subject to the restriction of the LBB condition. It can accommodate equal-order interpolations.

In order to show the properties of the least-squares finite element method for first-order systems, we include an error analysis and numerical experiments for a simple one-dimensional problem.

In the following pages the least-squares method is introduced through a simple one-dimensional problem and then an error analysis for this simple problem is presented. The velocity-pressure-vorticity formulation for the Stokes problem is described, followed by an introduction to the least-squares finite element method for the stokes problem. Numerical results showing the optimal rate of convergence are then given.

\section{ONE-DIMENSIONAL PROBLEM}

In order to show the difference between the Galerkin mixed method and the least-squares method, we introduce here a simple one-dimensional problem as

$$
\begin{array}{cc}
\frac{d^{2} u}{d x^{2}}=f & \text { in }(0,1) \\
u(0)=0 & \frac{d u}{d x}(1)=0
\end{array}
$$

where $f \in L^{2}(0,1)$. It can be rewritten as the following first-order system:

$$
\begin{array}{cc}
\frac{d p}{d x}=f & \text { in }(0,1) \\
p-\frac{d u}{d x}=0 & \text { in }(0,1) \\
u(0)=0 & p(1)=0
\end{array}
$$

A weak form of the Galerkin method corresponding to equation (2) is given in reference 7 . Find $u \in H$ and $p \in S$, such that 


$$
\begin{array}{ll}
\int_{0}^{1}\left(p \frac{d v}{d x}+f v\right) d x=0 & \forall v \in H \\
\int_{0}^{1}\left(p-\frac{d u}{d x}\right) q d x=0 & \forall q \in S
\end{array}
$$

where $H=\left\{v \in H^{l}(0,1), v(0)=0\right\}$, and $S=\left\{q \in L^{2}(0,1)\right\}$.

One can construct the finite element method based on equation ( 3 ), but the interpolation of $u$ and $p$ cannot be chosen independently. To assure the existence of the discrete solutions, the following LBB condition must be satisfied:

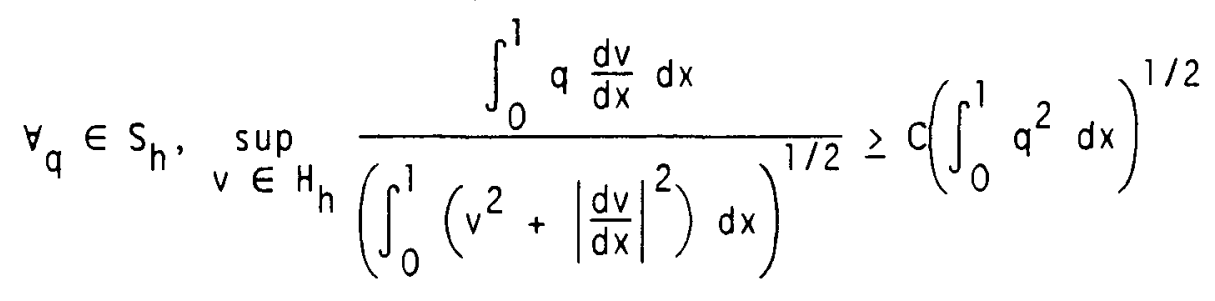

where $H_{h}$, $S_{h}$ denote the corresponding finite element subspace. If we choose $H_{h}=$ set of piecewise linear continuous functions and $\mathrm{S}_{h}=$ set of piecewise constant functions, then the rate of convergence is

$$
\left(\int_{0}^{1}\left(\left|u-u_{h}\right|^{2}+\left|\frac{d}{d x}\left(u-u_{h}\right)\right|^{2}+\left|p-p_{h}\right|^{2}\right) d x\right)^{1 / 2} \leq c h
$$

Now let us consider a least-squares method for equation (2). We construct a least-squares functional

$$
\begin{gathered}
J: X \times H \rightarrow R \\
J(p, u)=\frac{1}{2} \int_{0}^{1}\left[\left(\frac{d p}{d x}-f\right)^{2}+\left(p-\frac{d u}{d x}\right)^{2}\right] d x
\end{gathered}
$$

where $x=\left\{q \in H^{1}(0,1) ; q(1)=0\right\}$. Taking the variation with respect to $p$ and $u$, and letting $\delta J=0$ lead to a least-squares weak statement, find $U=(p, u) \in X \times H$ such that

$$
\begin{gathered}
\int_{0}^{1}\left[\frac{d p}{d x} \frac{d q}{d x}+\left(p-\frac{d u}{d x}\right)\left(q-\frac{d v}{d x}\right)\right] d x=\int_{0}^{1} f \frac{d q}{d x} d x \\
\forall V=(q, v) \in X \times H
\end{gathered}
$$

The corresponding finite element problem is then to find $U_{h}=\left(p_{h}, u_{h}\right) \in X_{h} \times H_{h}$ such that

$$
B\left(U_{h}, V_{h}\right)=L\left(V_{h}\right) \quad \forall V_{h}=\left(q_{h}, v_{h}\right) \in X_{h} \times H_{h}
$$


where

$$
\begin{gathered}
B\left(U_{h}, v_{h}\right)=\left(\frac{d p_{h}}{d x}, \frac{d q_{h}}{d x}\right)+\left(p_{h}-\frac{d u_{h}}{d x}, q_{h}-\frac{d v_{h}}{d x}\right) \\
L\left(v_{h}\right)=\left(f, \frac{d q_{h}}{d x}\right)
\end{gathered}
$$

in which $(\cdot, \cdot)$ denotes the $L^{2}$ inner product and $h$ is the mesh parameter.

\section{ERROR ANALYSIS FOR ONE-DIMENSIONAL PROBLEM}

It is easy to verify that

$$
B(U, V) \leq C\|U\|_{1} \cdot\|V\|_{1}
$$

where $\|u\|_{1}^{2}=\|p\|_{1}^{2}+\|u\|_{1}^{2}$, in which $\|\cdot\|_{1}$ denotes the $H^{1}$ norm. Thus, $B(\cdot, \cdot)$ is continuous on $X \times H . B(\cdot, \cdot)$ is symmetric and the inequalities in the Lax-Milgram theorem reduce to the single coercivity requirement. There exists a constant $\alpha>0$ such that for $V \in X \times H$

$$
B(V, V) \geq \alpha\|V\|_{1}^{2}
$$

In fact,

$$
B(V, v)=\left\|\frac{d q}{d x}\right\|^{2}+\left\|q-\frac{d v}{d x}\right\|^{2}
$$

in which $\|\cdot\|$ denotes the $L_{2}$ norm. Consequently,

$$
\begin{gathered}
B(V, V) \geq\left\|\frac{d q}{d x}\right\|^{2} \\
B(V, V) \geq\left\|q-\frac{d V}{d x}\right\|^{2}
\end{gathered}
$$

From equation (13) we have

$$
\begin{aligned}
B(V, v) & =\left\|\frac{d q}{d x}\right\|^{2}+\|q\|^{2}+\left\|\frac{d v}{d x}\right\|^{2}-2\left(q, \frac{d v}{d x}\right) \\
& \geq\left\|\frac{d q}{d x}\right\|^{2}+\|q\|^{2}+\|v\|^{2}+2\left(\frac{d q}{d x}, v\right)
\end{aligned}
$$


(by integration-by-parts and the boundary conditions in equation (2), and by the Friedrichs inequality $\left\|\frac{d v}{d x}\right\|^{2} \geq\|v\|^{2}, \forall v \in H$ ). So

$$
B(V, v) \geq\left\|\frac{d q}{d x}+v\right\|^{2}+\|q\|^{2}
$$

From equation (16)

$$
\begin{gathered}
B(V, V) \geq\left\|\frac{d q}{d x}+v\right\|^{2} \\
B(V, V) \geq\|q\|^{2}
\end{gathered}
$$

The combination of equations (14) and (17) leads to

$$
2(B(V, v))^{1 / 2} \geq\left\|\frac{d q}{d x}\right\|+\left\|\frac{d q}{d x}+v\right\|=\left\|-\frac{d q}{d x}\right\|+\left\|\frac{d q}{d x}+v\right\| \geq\|v\|
$$

so that

$$
4 B(V, V) \geq\|v\|^{2}
$$

Similarly, the combination of equations (15) and (18) leads to

$$
2(B(v, v))^{1 / 2} \geq\left\|q-\frac{d v}{d x}\right\|+\|q\|=\left\|\frac{d v}{d x}-q\right\|+\|q\| \geq\left\|\frac{d v}{d x}\right\|
$$

so that

$$
4 B(V, v) \geq\left\|\frac{d v}{d x}\right\|^{2}
$$

By combining equations (14), (18), (19), and (20) together, we obtain the coercivity

$$
B(V, V) \geq \frac{1}{10}\left(\|q\|_{1}^{2}+\|v\|_{1}^{2}\right)=\frac{1}{10}\|v\|_{1}^{2}
$$

Therefore, the following theorem about the rate of convergence of the corresponding finite element solutions can be proved directly (ref. 3 ).

Theorem. - Assume $f$ is smooth enough and the finite element interpolation estimates hold; that is,

$$
\begin{aligned}
& \left\|p-\Pi_{h} p\right\|_{l}^{2} \leq C_{p} h^{2 l} \\
& \left\|u-\Pi_{h} u\right\|_{l}^{2} \leq C_{u} h^{2 m}
\end{aligned}
$$


then

$$
\left\|p-p_{h}\right\|_{1}^{2}+\left\|u-u_{h}\right\|_{1}^{2} \leq c\left(h^{2 l}+h^{2 m}\right)
$$

where $\Pi_{h}$ denotes the finite element interpolation, $\ell$ and $m$ denote the orders of polynomials for $p$ and $u$, respectively, and $C_{p}, C_{u}$, and $C$ are the constants which do not depend on the mesh size $h$.

We may utilize the Aubin-Nitsche trick to obtain the optimal $L^{2}$ estimates of the error

$$
\begin{aligned}
& \left\|u-u_{h}\right\| \leq C h^{k+1} \\
& \left\|p-p_{h}\right\| \leq C h^{k+1}
\end{aligned}
$$

where $k=\min (\ell, m)$.

From the above procedure, we know that the boundary conditions play an important role in guaranteeing the coercivity. Now let us consider the same problem with different boundary conditions as follows:

$$
\begin{array}{ll}
\text { Case (a) } u(0)=0, & u(1)=0 \\
\text { Case (b) } u(0)=0, & p(0)=0
\end{array}
$$

In case (a), more boundary conditions are given for one variable, and no boundary condition is prescribed for the other variable. A similar situation is found in the Stokes problem, where more boundary conditions are related to velocity, and less or no boundary conditions are known for pressure and vorticity. This makes the verification of solvability of the first-order systems nontrivial.

In case (a), we may follow the same procedure as before to prove the coercivity of the corresponding bilinear form $B(V, V)$.

In case (b), we still have equations (14) and (15). From equation (14) and the Friedrichs inequality $\|d q / d x\|^{2} \geq\|q\|^{2}, \forall q \in H$, we have

$$
B(V, V) \geq\|q\|^{2}
$$

The combination of equations (15) and (26) leads to

$$
2(B(V, V))^{1 / 2} \geq\|q\|+\left\|-q+\frac{d v}{d x}\right\| \geq\left\|\frac{d v}{d x}\right\|
$$

so that

$$
4 B(V, V) \geq\left\|\frac{d v}{d x}\right\|^{2}
$$


From equation (27) and the Friedrichs inequality for $\forall v \in H$, we have

$$
4 B(V, V) \geq\|v\|^{2}
$$

By combining equations (14), (26), (27), and (28) together, we obtain the coercivity; that is, for $V \in H \times H$

$$
B(V, V) \geq \frac{1}{10}\|V\|_{1}^{2}
$$

Remark 1. - The symmetry of the bilinear form $B(U, V)$ and the coercivity of $B \overline{(V, V)}$ guarantee that the matrix for the least-squares finite element method is symmetric positive definite. This is an important advantage of the least-squares method over the mixed methods.

Remark 2. - For the least-squares method, the choice of interpolations for $p$ and $u$ is not subject to any restriction as long as $\ell \geq 1, m \geq 1$. However, inspection of the second part of equation (2) shows that in order to make the residual of this equation equal to zero throughout, one may choose the interpolation for $u$ as one order higher than that for $p$.

To show the convergence of the least-squares finite element method, we performed numerical experiments for three simple, one-dimensional, boundaryvalue problems. We used a uniform mesh containing elements of length $h$. We are interested in the behavior of the error $e_{p}=p-p_{h}$ and $e_{u}=u-u_{h}$ in $L^{2}$ norm for various choices of polynomials of degree $l$ for $\mathrm{Ph}$ and $m$ for uh. Results of numerical experiments are shown in figure 1 and summarized in the following computed convergence rates for three model problems.

$$
\begin{aligned}
& \text { Problem 1: } u^{\prime}=p, p^{\prime}=-x^{3}, u(0)=u(1)=0 \\
& \qquad \begin{array}{r|r|r|r|}
\hline \text { Order } e & \text { Order } m & \left\|e_{p}\right\| & \left\|e_{u}\right\| \\
\hline 1 & 1 & 0\left(h^{2}\right) & 0\left(h^{2}\right) \\
2 & 1 & 0\left(h^{2}\right) & 0\left(h^{2}\right) \\
1 & 2 & 0\left(h^{2}\right) & 0\left(h^{2}\right) \\
2 & 2 & 0\left(h^{3}\right) & 0\left(h^{3}\right) \\
\hline \text { Problem 2: } u^{\prime}+3 u=p, p^{\prime}-2 u=-2 x^{2}+6 x-2, u(0)=u(1)=0
\end{array} \\
& \text { Problem 3: }-(a(x) u)^{\prime}=p, p^{\prime}=f, u(0)=u(1)=0 \\
& a(x)=\frac{1}{\alpha}+\alpha\left(x-x_{0}\right)^{2} \\
& f(x)=2+2 \alpha\left(x-x_{0}\right)\left\{\tan ^{-1}\left[\alpha\left(x-x_{0}\right)\right]+\tan ^{-1}\left(\alpha x_{0}\right)\right\} \\
& \alpha=0.5, x_{0}=0.5
\end{aligned}
$$

\begin{tabular}{|c|r|l|l|}
\hline Order $e$ & Order $m$ & $\left\|e_{p}\right\|$ & $\left\|e_{u}\right\|$ \\
\hline 1 & 1 & $0\left(h^{2}\right)$ & $0\left(h^{2}\right)$ \\
2 & 1 & $0\left(h^{2}\right)$ & $0\left(h^{2}\right)$ \\
1 & 2 & $0\left(h^{2}\right)$ & $0\left(h^{2}\right)$ \\
2 & 2 & $0\left(h^{3}\right)$ & $0\left(h^{3}\right)$ \\
\hline
\end{tabular}

\begin{tabular}{|c|c|c|l|}
\hline Order $e$ & Order $m$ & $\left\|e_{\mathrm{p}}\right\|$ & $\left\|e_{\mathrm{u}}\right\|$ \\
\hline 1 & 1 & $0\left(\mathrm{~h}^{2}\right)$ & $0\left(\mathrm{~h}^{2}\right)$ \\
2 & 2 & $0\left(\mathrm{~h}^{3}\right)$ & $0\left(\mathrm{~h}^{3}\right)$ \\
\hline
\end{tabular}


We obtained the rate of convergence of the method by calculating the norm of the error for each $h$, plotting log \|error\| versus $\log h$, and calculating the slope of this line. All of the computed rates of convergence agree with the estimate (eq. (24)). Of course, the error estimate for problem 2 must be proved theoretically.

\section{VELOCITY-PRESSURE-VORTICITY FORMULATION OF STOKES EQUATIONS}

Let us consider an application of the least-squares method to the Stokes equations. By introducing the vorticity $\underline{\omega}=$ curl $\underline{u}$ as an auxiliary variable, the Stokes equations can be written as

$$
\begin{gathered}
\text { div } \underline{u}=0 \\
v \text { curl } \underline{\omega}+\operatorname{grad} p=\underline{f} \\
\underline{\omega}-\operatorname{curl} \underline{u}=0
\end{gathered}
$$

where $\underline{u}$ is the velocity vector, $p$ is the pressure, $f$ is the body force vector, and $v$ is the viscosity. We shall consider the following two-dimensional problem only:

$$
\begin{gathered}
\frac{\partial u}{\partial x}+\frac{\partial v}{\partial y}=0 \\
\frac{\partial p}{\partial x}+v \frac{\partial \omega}{\partial y}=f_{x} \quad \text { in } \Omega \\
\frac{\partial p}{\partial y}+v \frac{\partial \omega}{\partial x}=f_{y} \\
\omega+\frac{\partial u}{\partial y}-\frac{\partial v}{\partial x}=0
\end{gathered}
$$

where $\omega$ denotes the $z$-component of $\omega,\left(f_{x}, f_{y}\right)$ is the body force vector, and $\Omega$ is a bounded domain in $R^{2}$ with piecewise smooth boundary $\Gamma$. For accommodating various combinations of boundary conditions, let $\left\{\Gamma_{1}, \Gamma_{2}, \Gamma_{3}, \Gamma_{4}, \Gamma_{5}\right\}$

denote the sides of $\Gamma$. The unit outward normal vector to $\Gamma$ is denoted by $\underline{n}$, and the tangential vector to $\Gamma$ is denoted by $\underline{t}$.

We can write equation (31) in the general form of a first-order system:

$$
\underline{L} \underline{u}=\underline{f}
$$

or

$$
A_{1} \frac{\partial \underline{u}}{\partial x}+A_{2} \frac{\partial \underline{u}}{\partial y}+B \underline{u}=f
$$


where

$$
\begin{array}{ll}
A_{1}=\left[\begin{array}{rrrr}
1 & 0 & 0 & 0 \\
0 & 0 & 1 & 0 \\
0 & 0 & 0 & -v \\
0 & -1 & 0 & 0
\end{array}\right] & A_{2}=\left[\begin{array}{llll}
0 & 1 & 0 & 0 \\
0 & 0 & 0 & v \\
0 & 0 & 1 & 0 \\
1 & 0 & 0 & 0
\end{array}\right] \\
B=\left[\begin{array}{llll}
0 & 0 & 0 & 0 \\
0 & 0 & 0 & 0 \\
0 & 0 & 0 & 0 \\
0 & 0 & 0 & 1
\end{array}\right] \quad \underline{f}=\left[\begin{array}{l}
0 \\
f_{f}^{x} \\
0
\end{array}\right] \quad \underline{u}=\left[\begin{array}{l}
u \\
v \\
p \\
\omega
\end{array}\right]
\end{array}
$$

The boundary conditions should be supplemented to complete the boundaryvalue problem. We may consider the following boundary conditions:

$$
\begin{array}{ll}
u, v & \text { given on } \Gamma_{1} \\
u_{n}, \omega & \text { given on } \Gamma_{2} \\
p, u_{t} & \text { given on } \Gamma_{3} \\
p, \omega & \text { given on } \Gamma_{4} \\
p, u_{n} & \text { given on } \Gamma_{5}
\end{array}
$$

For example, $\Gamma_{1}$ could be the inflow, outflow, or wall boundary; $\Gamma_{2}, \Gamma_{3}, \Gamma_{4}$, and $\Gamma_{5}$ could be the free surface, inflow, or outflow boundary.

The solvability of the boundary-value problem depends on the combination of the boundary conditions. For many practical problems, the boundary operator does not satisfy the Lopatinski condition (ref. 6). Thus, the proof of solvability involves a technical difficulty. We address this problem and give the error analysis in "An Error Analysis of Least-Squares Finite Element Method of Velocity-Pressure-Vorticity Formulation for Stokes Problem." (Technical Report, Cleveland State University, 1988.)

\section{LEAST-SQUARES FINITE ELEMENT FORMULATION FOR STOKES PROBLEM}

We first discretize the domain $\Omega$ as a union of finite elements and then introduce an appropriate finite element basis. Let $\mathrm{Ne}$ denote the number of nodes for one element, $\psi_{j}$ denote the element shape functions, and $u_{j}, v_{j}, p_{j}$, and $\omega_{j}$ be the nodal values. Equal-order interpolations are employed, so we can write the expansion

$$
\underline{u}_{h}(x, y)=\sum_{j=1}^{N e} \psi_{j}(x, y)\left[\begin{array}{l}
u_{j} \\
v_{j} \\
p_{j} \\
\omega_{j}
\end{array}\right]
$$


We define the least-squares functional

$$
\begin{gathered}
J:\left(H_{h}^{l}(\Omega)\right)^{4} \rightarrow R \\
J\left(\underline{u}_{h}\right)=\frac{1}{2}\left\|L \underline{u}_{h}-f\right\|^{2}=\frac{1}{2}\left\|A_{1} \frac{\partial \underline{u}_{h}}{\partial x}+A_{2} \frac{\partial \underline{u}_{h}}{\partial y}+B \underline{u}_{h}-f\right\|^{2}
\end{gathered}
$$

where each element in $\left(H_{h}^{l}(\Omega)\right)^{4}$ satisfies the boundary conditions.

By introducing the finite element approximation defined in equation (34) into the functional equation (35), we have the minimization problem as follows: find $\underline{u}_{h} \in\left(H_{h}^{1}(\Omega)\right)^{4}$, such that

$$
J\left(\underline{u}_{h}\right) \leq J\left(\underline{v}_{h}\right) \quad \forall \underline{v}_{h} \in\left(H_{h}^{l}(\Omega)\right)^{4}
$$

Obviously, this problem is equivalent to solving the aigebraic equations

$$
\mathrm{KU}=\mathrm{F}
$$

where the $U$ is the global vector of nodal values, the global matrix $K$ is assembled from the element matrices

$$
K_{e}=\int_{\Omega_{e}}\left(L \psi_{1}, L \psi_{2}, \ldots, L \psi_{N e}\right)^{\top}\left(L \psi_{1}, L \Psi_{2}, \ldots, L \psi_{N e}\right) d x d y
$$

and the vector $F$ is assembled from the element vector

$$
F_{e}=\int_{\Omega_{e}}\left(L \psi_{1}, L \psi_{2}, \ldots, L \psi_{N e}\right)^{\top} f d x d y
$$

in which

$$
\begin{aligned}
L_{i} & =\psi_{i}, x^{A}+\psi_{i}, y^{A}+\psi_{i} B \\
& =\left[\begin{array}{cccc}
\psi_{i}, x & \psi_{i}, y & 0 & 0 \\
0 & 0 & \psi_{i}, x & v \psi_{i}, y \\
0 & 0 & \psi_{i}, y & -v \psi_{i}, x \\
\psi_{i}, y & -\psi_{i}, x & 0 & \psi
\end{array}\right]
\end{aligned}
$$

and $T$ denotes the transposition. We observe that the matrix $K$ is symmetric positive definite.

As is customary, we use Gaussian quadrature to evaluate the coefficients of $\mathrm{K}_{e}$ and $\mathrm{F}_{\mathrm{e}}$. The number of "Gauss" points required for the solution is of 
some importance. Inspection of the fourth part of equation (31) shows that the vorticity $\omega$ and the derivatives of velocity components $u$ and $v$ appear simultaneously. When an equal-order interpolation is employed, it is impracticable to reduce the residual of this equation to zero throughout. For this reason, in our numerical experiments we use reduced integration (ref. 8 ).

\section{NUMERICAL RESULTS FOR STOKES PROBLEM}

We construct a model problem (ref. 9) corresponding to the polynomial divergence-free velocity field

$$
\begin{aligned}
& u(x, y)=x^{2}(1-x)^{2}\left(2 y-6 y^{2}+4 y^{3}\right) \\
& v(x, y)=y^{2}(1-y)^{2}\left(-2 x+6 x^{2}-4 x^{3}\right)
\end{aligned}
$$

the pressure field

$$
p(x, y)=x^{2}-y^{2}
$$

and the vorticity field

$$
\omega(x, y)=-x^{2}(1-x)^{2}\left(2-12 y+12 y^{2}\right)+y^{2}(1-y)^{2}\left(-2+12 x-12 x^{2}\right)
$$

with three groups of boundary conditions shown in figure 2 .

The bilinear element and the eight-node quadratic element were tested and uniform meshes were used. We employed one-point Gaussian quadrature for the bilinear element, and $2 \times 2$ quadrature for the eight-node quadratic element. The numerical results of the rate of convergence are shown in figure 3 . We found that in all tested cases

$$
\left\|e_{\underline{u}}\right\| \leq c h^{k+1} \quad\left\|e_{p}\right\| \leq c h^{k+1} \quad\left\|e_{\omega}\right\| \leq c h^{k+1}
$$

where $k$ is the order of polynomial of shape functions; that is, all variables $u, v, p$, and $\omega$ converge in $L_{2}$ norm at the optimal rate.

\section{CONCLUSIONS}

In contrast to the Galerkin mixed method based on the velocity-pressurevorticity formulation of the Stokes problem (refs. 7 and 10), the least-squares method presented here does not depend on the LBB condition. We have shown that the least-squares method converges at the optimal rate for equal-order interpolations. In contrast to the penalty method (refs. 11 and 12) and the PetrovGalerkin method (refs. 4 and 5 ), the least-squares method does not have any added parameters in the scheme. This means that the least-squares method is robust. In contrast to the methods based on the stream function-vorticity formulation (refs. 10 and 12), the least-squares method produces the velocity and pressure directly without further numerical calculation. This method can also be extended to three-dimensional cases.

The least-squares methods have been successfully applied to many problems, including high-speed flow with strong shocks (B.N. Jiang and G.F. Carey, "A Stable Least-Squares Finite Element Method for Nonlinear Hyperbolic Problems, 
Int. J. Numer. Meth. Fluids, to be published, and ref. 13). It is possible to make a general-purpose program based on the first-order system in order to attack various types of problems. To adapt the method to other problems, it is necessary only to modify the subroutines associated with the coefficient matrices $A_{1}, A_{2}$, and $B$ and the vector function $f$. The only disadvantage of the least-squares method is that each node has more variables.

The method presented here has already been extended to the incompressible Navier-Stokes equations and will be discussed in another paper.

\section{REFERENCES}

1. Babuška, I.: Error Bounds for Finite Element Method. Numer. Math., vol. 16, no. 4, 1971, pp. 322-333.

2. Brezzi, F.: On the Existence, Uniqueness and Approximation of SaddlePoint Problems Arising From Lagrange Multipliers. Rev. Fr. Automatique Inform. Rech. Opér., vol. 8, Aug. 1974, pp. 129-151.

3. Oden, J.T.; and Carey, G.F.: Finite Elements: Mathematical Aspects, Vol. IV. Prentice-Hall, 1983.

4. Hughes, T.J.R.; Franca, L.P.; and Balestra, M.: A New Finite Element Formulation for Computational Fluid Dynamics: V. Circumventing the Babuska-Brezzi Condition: A Stable Petrov-Galerkin Formulation of the Stokes Problem Accommodating Equal-Order Interpolations. Comput. Methods App 1. Mech. Eng., vol. 59, no. 1, Nov. 1986, pp. 85-99.

5. Hughes, T.J.R.; and Franca, L.P.: A New Finite Element Formulation for Computational Fluid Dynamics: VII. The Stokes Problem With Various WellPosed Boundary Conditions: Symmetric Formulations that Converge for all Velocity/Pressure Spaces. Comput. Methods Appl. Mech. Eng., vol. 65, no. I, Nov. 1987, pp. 85-96.

6. Wendland, W.L.: Elliptic Systems in the Plane. Pitman Publishing Ltd., London, 1979.

7. Thomasset, F.: Implementation of Finite Element Methods for Navier-Stokes Equations. Springer-Verlag, New York, 1981.

8. Zienkjewicz, O.C.; Owen, D.R.J.; and Lee, K.N.: Least Squares Finite Element for Elasto-Static Problems - Use of Reduced Integration. Int. J. Numer. Methods Eng., vol. 8, no. 2, 1974, pp. 341-358.

9. Oden, J.T.; and Jacquotte, O.P.: Stability of Some Mixed Finite Element Methods for Stokesian Flows. Comput. Methods Appl. Mech. Eng., vol. 43, no. 2, 1984, pp. 231-247.

10. Girault, V.; and Raviart, P.A.: Finite Element Methods for Navier-Stokes Equations. Springer-Verlag, Berlin, 1986. 
11. Oden, J.T.; Kikuchi, N.; and Song, Y.J.: Penalty Finite Element Methods for the Analysis of Stokesian Flows. Comput. Methods Appl. Mech. Eng., vol. 31, no. 3, Aug. 1982, pp. 297-329.

12. Carey, G.F.; and Oden, J.T.: Finite Elements: Fluid Mechanics, Vol. VI. Prentice-Hall, 1986.

13. Jiang, B.N.; and Carey, G.F.: Least-Squares Finite Elements for Compressible Euler Equations. Numerical Methods in Laminar and Turbulent Flow, Vol. 5, C. Taylor, W.G. Habashi, and M.M. Hafez, eds., Pineridge Press, Swansea, U.K., 1987, pp. 1460-1464.

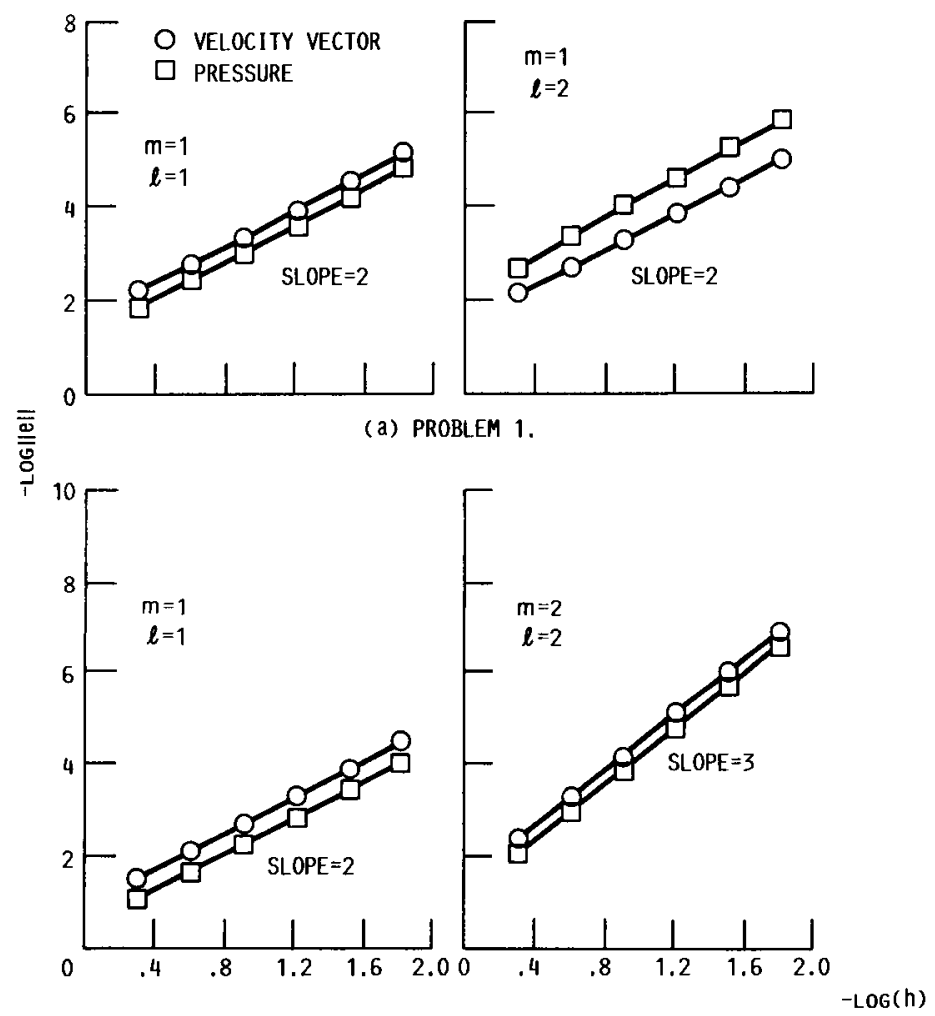

(c) PROBLEM 2.

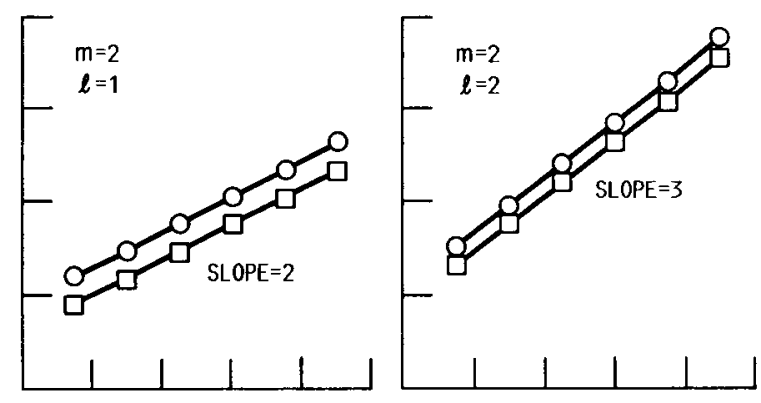

(b) PROBLEM 1 .

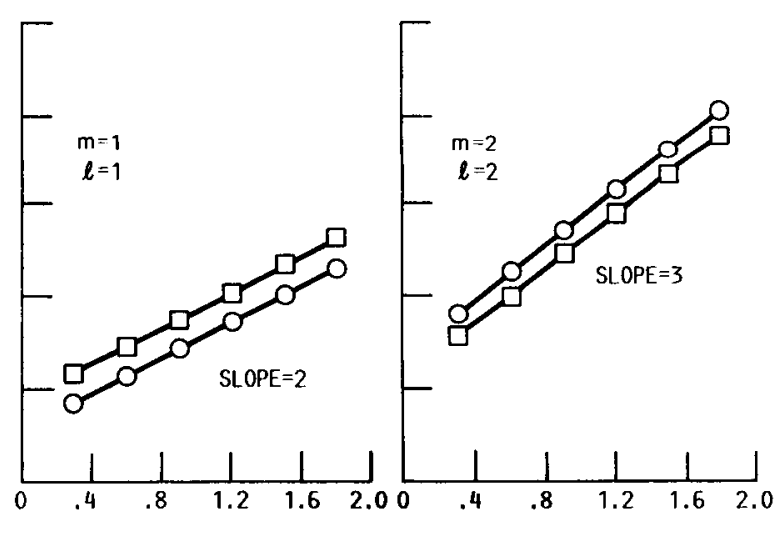

(d) PROBLEM 3.

FigURE 1. - COMPUTED CONVERGENCE RATE FOR ONE-DIMENSIONAL PROBLEMS. ORDER OF POLYMOMIALS FOR $p$ AND $u, l$ AND $m$. 


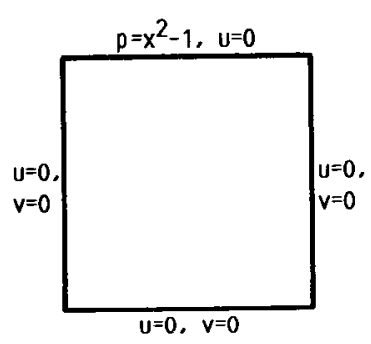

(a)

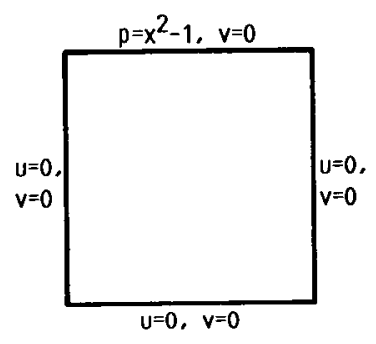

(b)

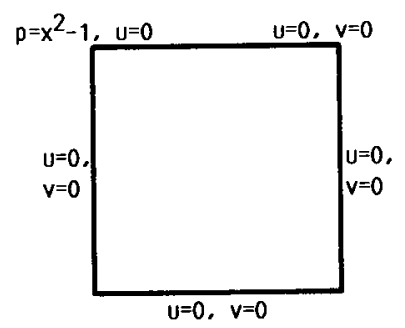

(c)

FIGURE 2. - BOUNDARY CONDITIONS FOR STOKES PROBLEM. 


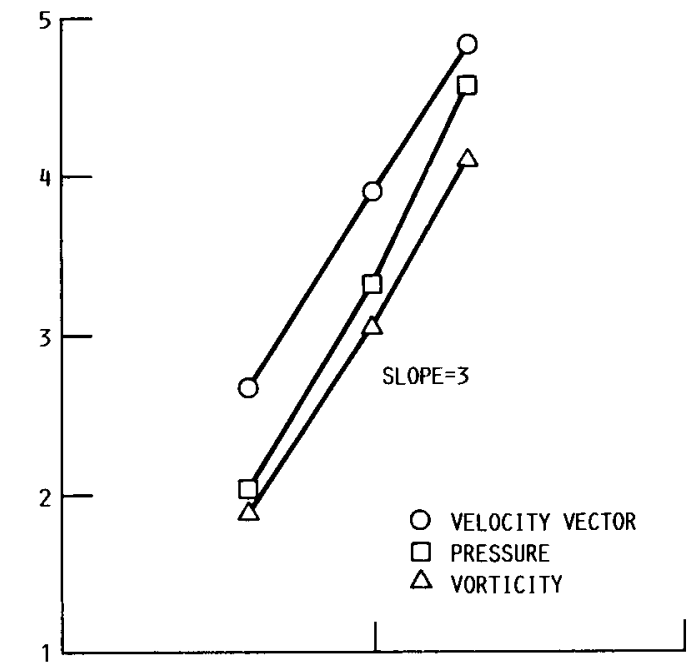

흥

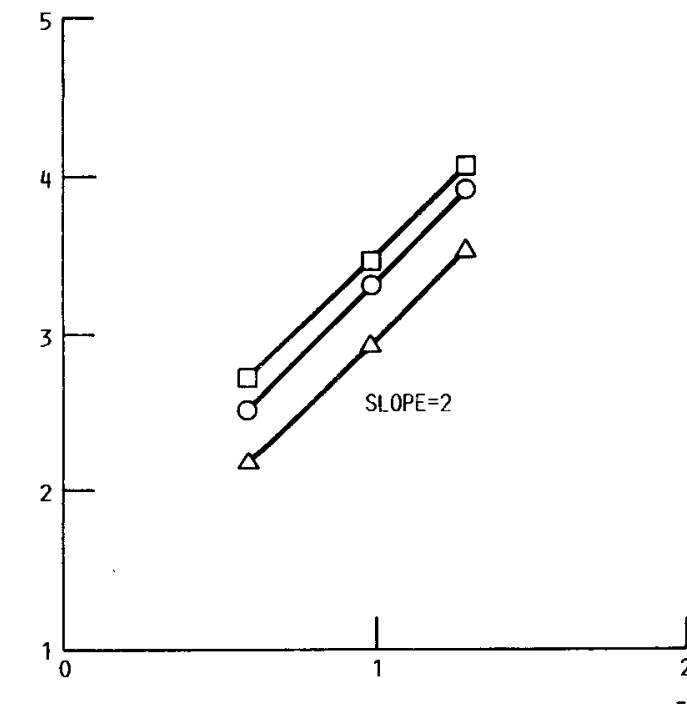

(c) BILINEAR ELEMENT FOR BOUNDARY CONDITION (b) FROM FIGURE 2.

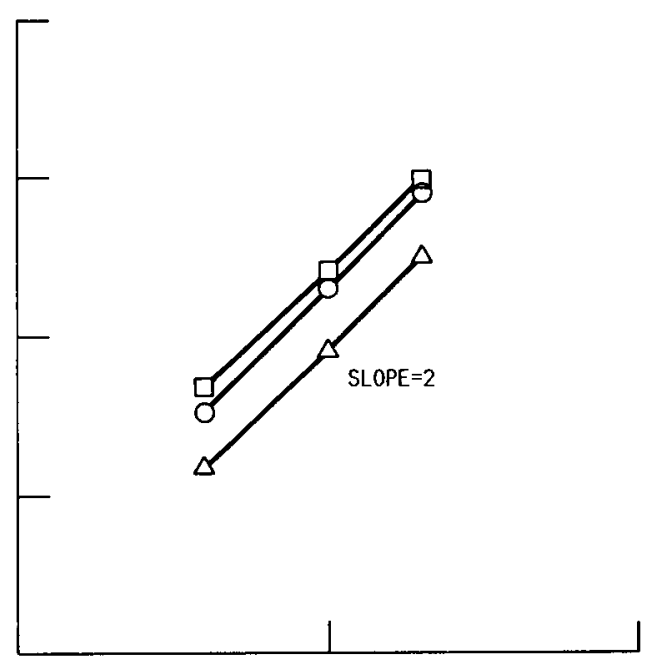

(b) BILINEAR ELEMENT FOR BOUNDARY CONDITION (a) FROM FIGURE 2.

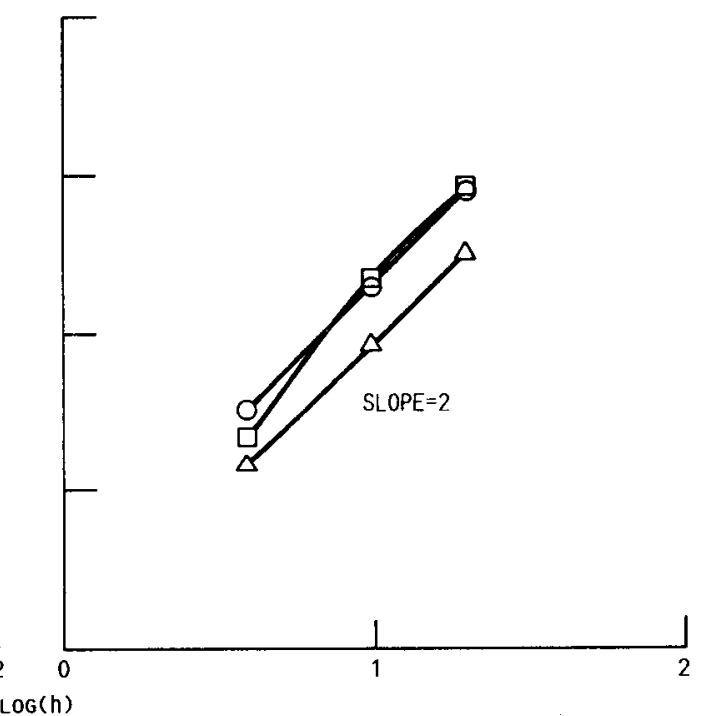

(d) BILINEAR ELEMENT FOR BOUNDARY CONDITION (c) FROM FIGURE 2.

FIGURE 3. - COMPUTED CONVERGENCE RATE FOR STOKES PROBLEM. 


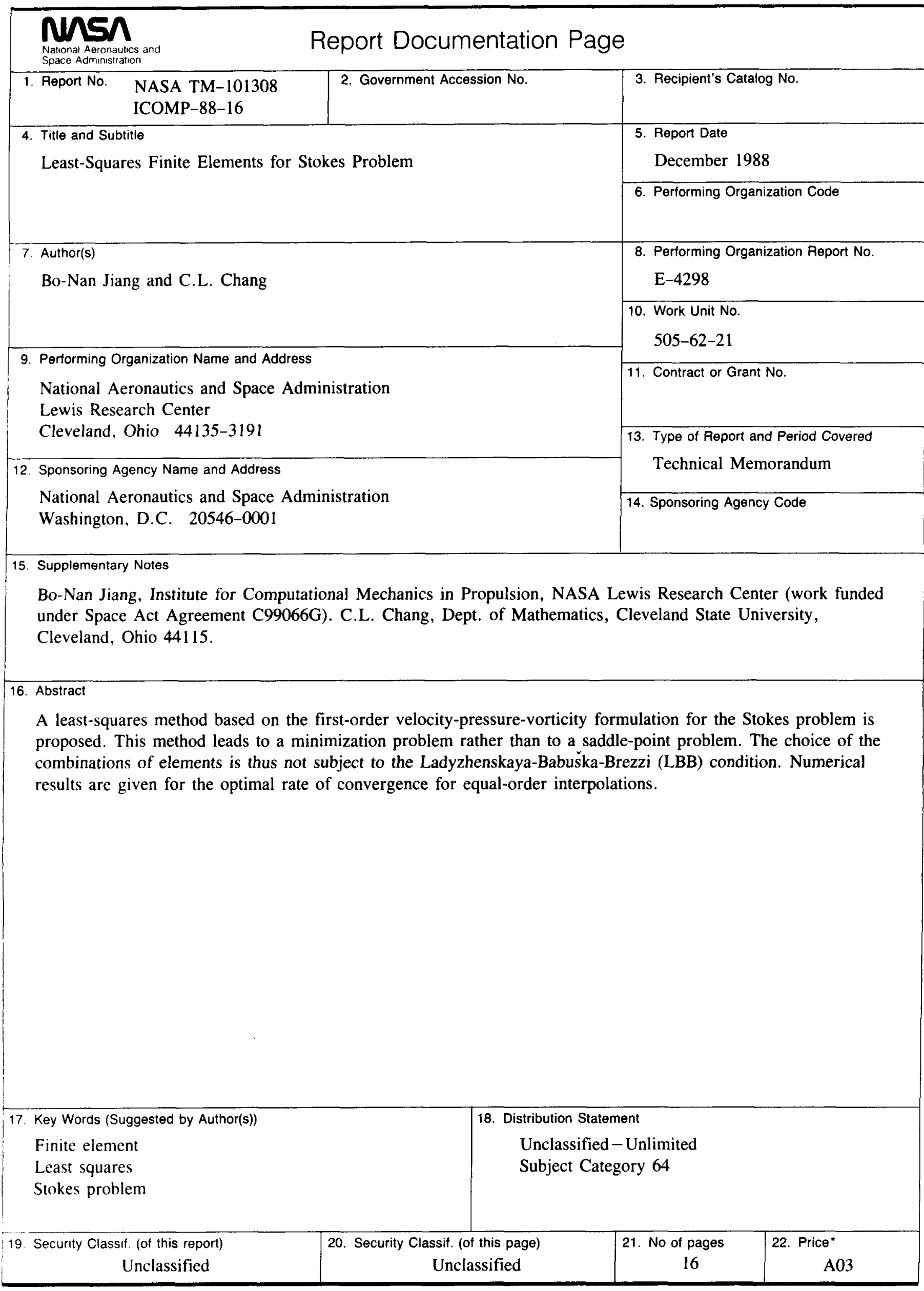

